
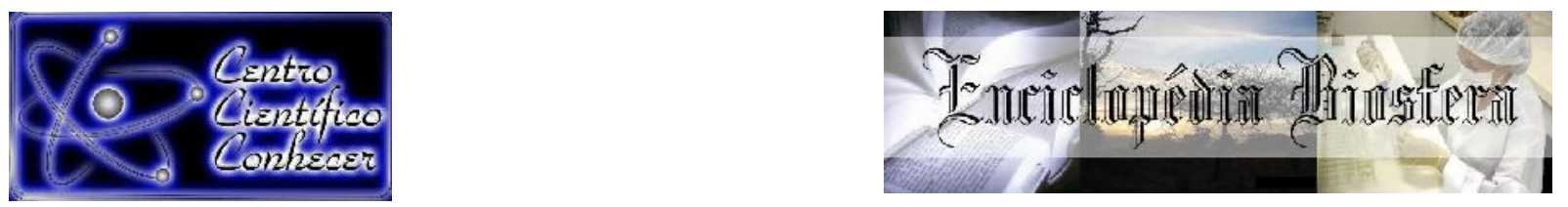

\title{
CRESCIMENTO EM DIÂMETRO E ALTURA DOMINANTE EM PLANTIOS CLONAIS DE Tectona grandis Linn F. SOB DOIS ESPAÇAMENTOS
}

\author{
Mario Lima dos Santos ${ }^{1}$; Richard Pinheiro Rodrigues²; Cássio Rafael Costa dos \\ Santos $^{2}$; Beatriz Cordeiro Costa ${ }^{3}$; Welton dos Santos Barros ${ }^{4}$ \\ ${ }^{1}$ Mestre em Ciências Florestais-Universidade Federal Rural da Amazônia, Belém, Brasil. \\ (mariolimaeng@gmail.com) \\ ${ }^{2}$ Mestres em Ciências Florestais - Universidade Federal Rural da Amazônia. \\ ${ }^{3}$ Graduanda de Engenharia Florestal - Universidade Federal Rural da Amazônia \\ ${ }^{4}$ Graduando de Engenharia Florestal - Universidade Federal Rural da Amazônia
}

Recebido em: 22/09/2018 - Aprovado em: 23/11/2018 - Publicado em: 03/12/2018 DOI: 10.18677/EnciBio_2018B29

\begin{abstract}
RESUMO
A espécie florestal Tectona grandis Linn. F., apresenta grande importância no setor florestal, sendo empregada para diferentes usos, como fabricação de móveis, elementos estruturais, laminados e pisos. Por este fato, torna-se necessário o estudo do ritmo de crescimento de suas variáveis dendrométricas, para servir de base ao planejamento florestal. Dessa forma, o presente estudo teve por objetivo avaliar o crescimento em diâmetro e altura dominante sob dois espaçamentos em plantios clonais de $T$. grandis nas idades de cinco e sete anos. O trabalho foi realizado no Estado do Pará, município de Capitão Poço. O levantamento foi realizado em 43 parcelas permanentes, distribuídas sistematicamente na área. As variáveis coletadas foram: diâmetro a 1,30 m do solo (dap) e altura dominante $(H d)$. Foram analisados dois espaçamentos, $4 \mathrm{~m} \times 4 \mathrm{~m}$ (tratamento 1) e 3,75m x 3,75m (tratamento 2). Nos resultados, notou-se um maior crescimento em $\mathrm{Hd}$ no tratamento 2, relacionado ao menor espaçamento entre indivíduos. A prática silvicultural de desbaste afetou mais fortemente 0 crescimento em diâmetro, com a altura dominante sofrendo menor influência. O incremento médio anual em diâmetro variou de 3,4 a $7,0 \mathrm{~cm}^{2} \mathrm{ano}^{-1}$ nos dois tratamentos, em idades de um a sete anos, com resultados superiores ao crescimento em de plantios seminais comparados. O mesmo ocorreu com a altura dominante, obtendo-se IMA de 2,5 a 7,9 cm ano ${ }^{-1}$ até o sétimo ano. Os resultados possibilitam um melhor planejamento de manejo, subsidiando a condução dos plantios da espécie estudada.
\end{abstract}

PALAVRAS-CHAVE: Prática silvicultural, Incremento anual, Planejamento florestal, Variáveis dendrométricas. 


\title{
GROWTH FOR DOMINANT HEIGHT AND DIAMETER IN CLONAL PLANTINGS OF Tectona grandis Linn F. UNDER TWO SPACINGS
}

\begin{abstract}
The forest species Tectona grandis Linn. F., known as Teak, has great importance in forest sector, used for different ways, as manufacture of furniture, structural elements, laminates and floors. The studies regarding to growth rate from dendrometric variables of forest species plays an important role, essential to forestry planning. The purpose of this research was to evaluate the growth in diameter and dominant height from two different spacings from clonal plantings of Tectona grandis Linn. $\mathrm{F}$ between five and seven years of implantation. The study was performed in municipality of Capitão Poço, State of Pará, Brazil. The monitoring of clonal plantings was provided by continuous inventory of 43 circular plots with systematically distribution into a grid of $320 \times 320 \mathrm{~m}$. The dendrometrical variables collected were: dominant height $(H d)$ and diameter at breast heigt, over $1.30 \mathrm{~m}$ from soil (dap). There was the strongest growth of $\mathrm{Hd}$ in 2012 and 2013 clonal plantings in comparison with 2010, with regard to smaller spacing between individuals. The thinning strongly affected the growth in diameter, and dominant height were less influence. The annual average capacity in dap varied between 3.4 to $7.0 \mathrm{~cm}_{\text {year }}{ }^{-1}$ in both treatments, in the period of one to seven years, exceeded the growth results obtained from others investigations of seminal plantation. The same happened with the variable $H d$, obtaining IMA of 2.5 to $7.9 \mathrm{~cm}_{\text {year }}{ }^{-1}$ until the seventh year. This results allow a better management planning and taking of decisions, supporting the management in clonal plantations of $T$. grandis.
\end{abstract}

KEYWORDS: Silvicultural operation, Annual increase, Forestry planning, Dendrometrical variables.

\section{INTRODUÇÃO}

A espécie florestal Tectona grandis Linn. F., conhecida vulgarmente como teca, apresenta grande importância no setor florestal, tanto no mercado interno como externo (IBÁ, 2017). Midgley et al. (2015) estimaram que existem cerca de 6,8 milhões de hectares (ha) de plantios de teca no mundo, dos quais $88,2 \%$ estão na Ásia, $7,8 \%$ na África, $4 \%$ na América tropical. No Brasil, ela é plantada comercialmente nos estados do Acre, Amazonas, Mato Grosso e Pará, com área de 80 mil ha de plantios (IBÁ, 2017), sendo empregada para diferentes usos, como fabricação de móveis, elementos estruturais, laminados e pisos. Além disso, sua madeira também possui alta resistência a produtos químicos, sendo ideal para utilização de móveis (SILVA et al., 2016).

Neste contexto, para produção de madeira, torna-se necessário conhecer o ritmo de crescimento das variáveis dendrométricas a nível de povoamento, tendo em vista que essas informações influenciam nas decisões e na forma de como ocorrerá o manejo da floresta (MACHADO et al., 2015). Tais decisões, exigem informações referentes ao estoque futuro dos povoamentos, como o momento ideal para o primeiro desbaste e corte final, destino e venda da madeira (NASCIMENTO et al., 2015).

O conhecimento das variáveis altura dominante e diâmetro, possibilita identificar as relações entre a produção, por meio do incremento corrente anual $($ ICA) e do incremento médio anual (IMA) (MACHADO et al., 2015). A altura dominante é a variável 
que menos sofre influência quando o povoamento passa por tratamentos silviculturais como desbastes naturais e planejados (CAMPOS ; LEITE, 2017). No entanto, o diâmetro é mais suscetível a sofrer influência de fatores como a idade, genética, desbaste e espaçamento (ELOY et al., 2016; SILVA et al., 2016; MOULIN et al., 2017).

Hakamada et al. (2015), em um plantio florestal, mais precisamente, em um plantio clonal, constataram que todas as plantas possuem a mesma capacidade de crescimento, facilitando pesquisas e manejos. No Brasil, ainda há um desenvolvimento recente de programas genéticos que envolvam a teca registrando o melhoramento genético da espécie por meio de testes de progênies, clones e testes de procedência (GEORGIN et al., 2014), já existindo plantios em escala comercial que ainda não chegaram a sua rotação final. É em função do genótipo da espécie e especificidades do sítio que o espaço para desenvolvimento das árvores será definido, influenciando no desbaste, na qualidade da madeira que será produzida, na forma do tronco e na idade de corte (MEDEIROS et al., 2017).

Desse modo, o trabalho teve como objetivo avaliar o crescimento em diâmetro e altura dominante sob dois espaçamentos em plantios clonais de Tectona grandis Linn. F nas idades de cinco e sete anos, no município de Capitão Poço, Estado do Pará.

\section{MATERIAL E MÉTODOS}

\section{Área de estudo}

O trabalho foi conduzido em povoamentos clonais de T. grandis em 414,66 há, localizado no município de Capitão Poço, Estado do Pará, na empresa Tietê Agrícola LTDA (Figura 1), tendo área total de 2.399,62 ha, sitiada entre as coordenadas geográficas $2^{\circ} 30^{\prime} 00^{\prime \prime}$; 4 4020’00”W e 2²0’0”S; 47³0’0”W.

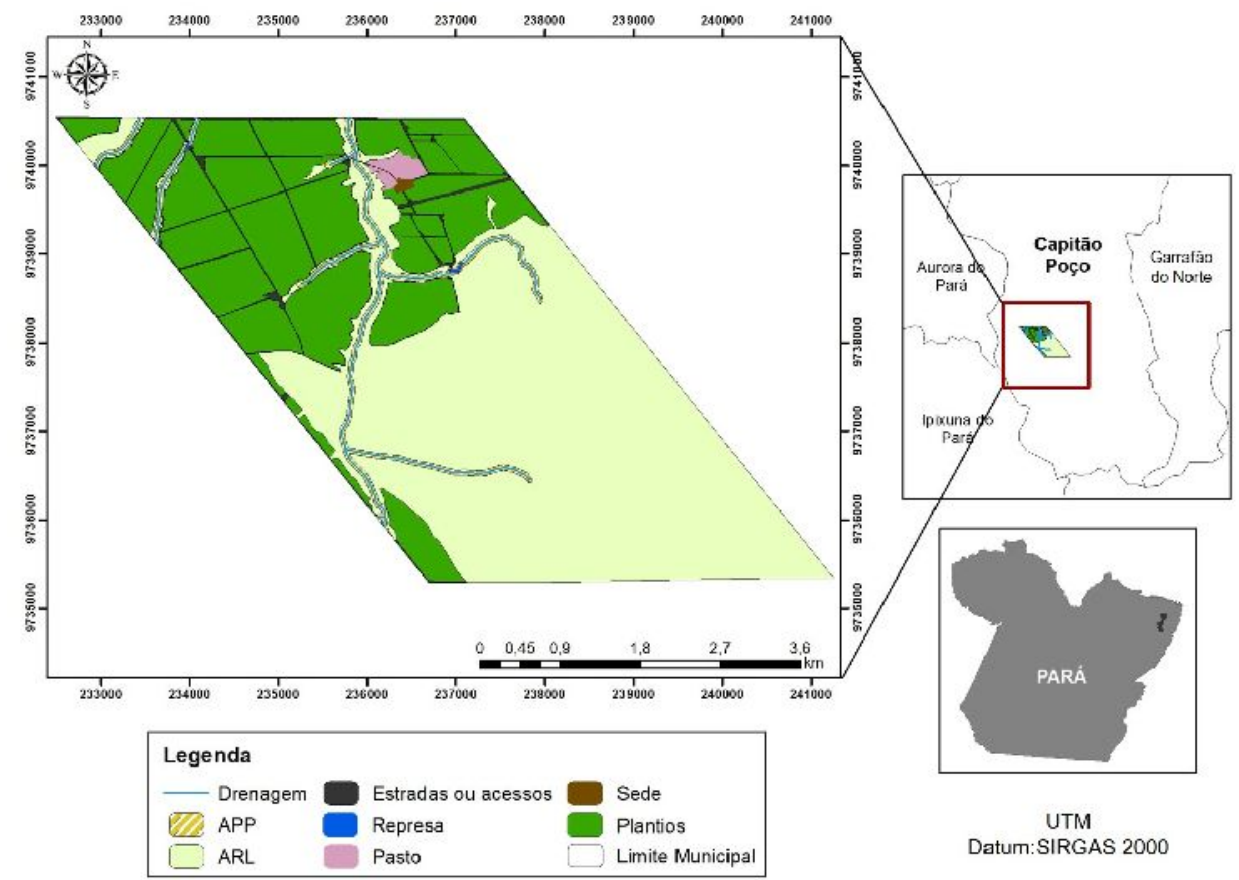

FIGURA 1. Localização da área de estudo, em Capitão Poço, PA. FONTE: Autores (2018). 
A vegetação é caracterizada por floresta equatorial subperenifolia, com grande parte da vegetação alterada remanescendo floresta explorada. O relevo é plano a suave ondulado, sendo que poucas áreas podem ser classificadas como de relevo ondulado ocupadas apenas com pastagem, e altitudes em torno de 79 metros (SILVA, 1999). De acordo com a classificação do Sistema Brasileiro de Classificação de Solos (SANTOS, 2013), a área de estudo possui predominância de Latossolo Amarelo Distrófico.

Os plantios foram realizados nos anos de 2010, 2012 e 2013, com espaçamentos de $4 \mathrm{~m} \times 4 \mathrm{~m}$ (plantio de 2010) e 3,75m x 3,75m (plantios de 2012 e 2013), onde formaram dois tratamentos $\left(T_{1}\right.$ e $\left.T_{2}\right)$. Estes tratamentos, tiveram atividades de plantio e manutenção semelhantes, obedecendo a sequência operacional (Tabela 1 ), conforme informações fornecidas pela empresa. Ao quinto ano no plantio 2010, foi realizado um desbaste seletivo com intensidade de retirada de $50 \%$ da área basal.

TABELA 1. Lista de atividades operacionais silviculturais e suas características implantadas nos plantios clonais de Tectona grandis Linn F. na fazenda São Luiz, em Capitão Poço, PA.

\begin{tabular}{llc}
\hline \multicolumn{1}{c}{ Atividade operacional } & \multicolumn{1}{c}{ Características das atividades } & Ano \\
\hline \hline Combate à formiga & Com iscas formicidas & 0 \\
Limpeza da área & Mecanizada & 0 \\
Calagem & 4 toneladas de calcário por hectare & 0 \\
Plantio & Manual & 1 \\
Replantio & Manual (>10\% de perda) & 1 \\
Adubação de arranque & NPK, KCl & 1 \\
Coroamento & Enxada & 1 \\
Roçagem & Semi e mecanizada & 1 ao 7 \\
Desrama & Serra e Motopoda & 1 ao 7 \\
Adubação de Manutenção & Boro, KCl & $1,3,5$ e 7 \\
Desbaste & Semi-mecanizado & 5 \\
\hline
\end{tabular}

FONTE: Autores (2018).

\section{Coleta dos dados}

O monitoramento dos plantios foi realizado por meio de inventário florestal em parcelas permanentes de 2011 à 2017 nos meses de agosto, com sistema de amostragem composto pelo método de área fixa, com parcelas circulares com raio de 12,61 metros cada, com uma área de aproximadamente $500 \mathrm{~m}^{2}$. O processo de amostragem empregado foi o sistemático, em que as parcelas foram distribuídas em uma grade de $320 \mathrm{~m} \times 320 \mathrm{~m}$ aplicada a todos os plantios (Figura 2). A distância entre os plantios dos tratamentos varia de $300 \mathrm{~m}$ à $3500 \mathrm{~m}$. 


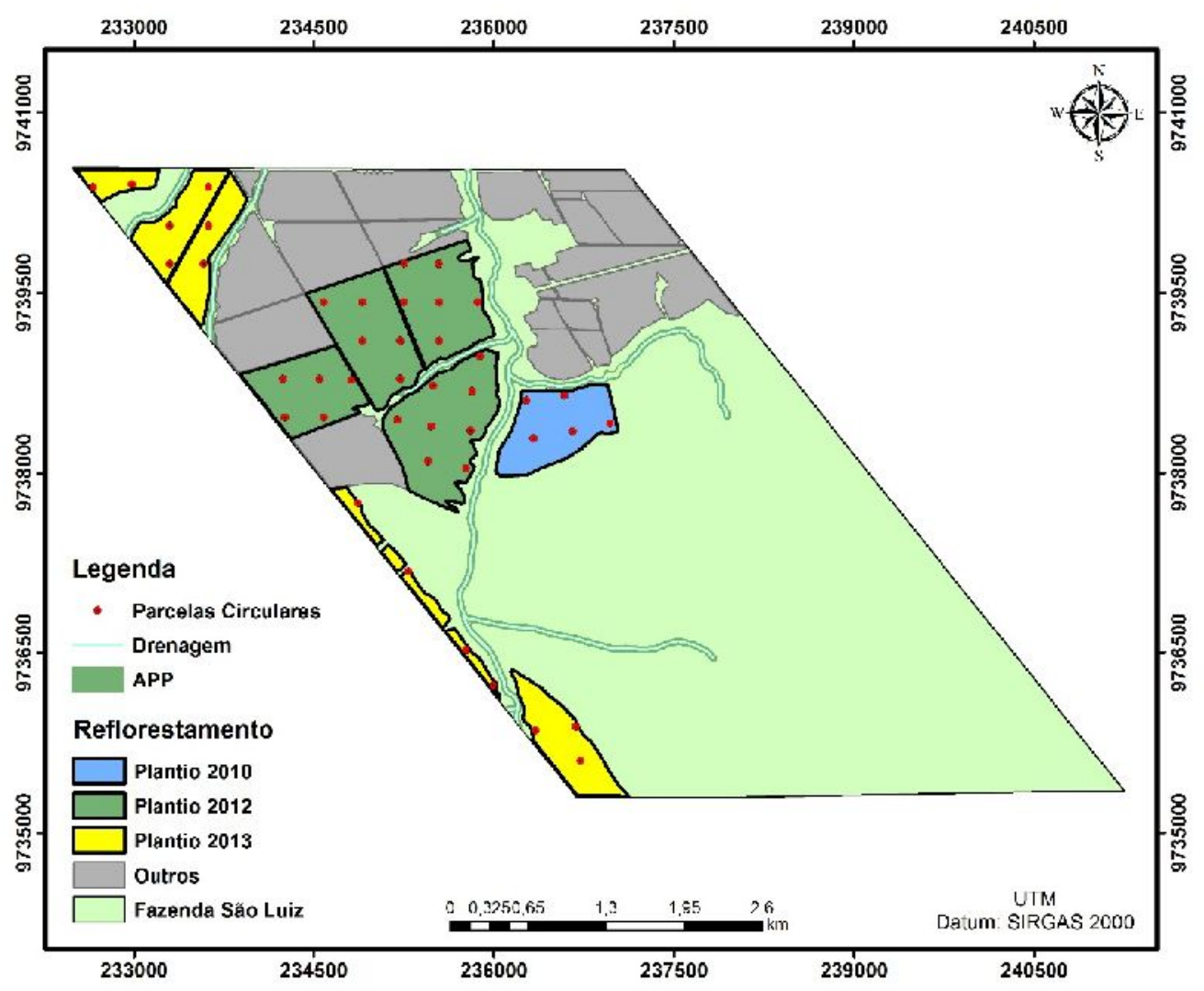

FIGURA 2. Mapa da fazenda São Luiz com distribuição das parcelas amostrais por idade dos plantios clonais de T. grandis em Capitão Poço, PA.

FONTE: Autores (2018).

As variáveis dendrométricas coletadas foram: Altura total $(H)$ em metros e diâmetro a $1,30 \mathrm{~m}$ do solo (dap) em centímetros. Ainda no campo, com dados de dap, foram identificados os indivíduos dominantes com suas respectivas alturas dominantes (Hd), seguindo a definição de Assmann (1970), disposto em Scolforo (1993), resultando em cinco árvores dominantes por parcela. Os instrumentos utilizados para a mensuração das variáveis foram: Hipsômetro Vertex para a altura total, fita diamétrica para o dap, receptor GPS (Garmim).

\section{Análise de dados}

Os dados de altura dominante $(H d)$ foram submetidos à análise de normalidade pelo teste de Kolmogorov-Smirnov a $5 \%$ de significância. Para verificar se houve diferença estatística em $\mathrm{Hd}$ entre os tratamentos $\mathrm{T}_{1}$ e $\mathrm{T}_{2}$ aplicou-se o teste t student a $95 \%$ de confiança, utilizando dados ao quinto ano para comparação.

A intensidade amostral $(n)$ foi calculada para verificar se o número de amostras utilizadas, estimou de maneira satisfatória a altura dominante para um dado intervalo de confiança, a 95\% de nível de probabilidade e erro máximo admissível de 10\%. A determinação deu-se de acordo com Pellico Netto e Brena (1997) para população 
infinita. Os cálculos do Incremento Corrente Anual (ICA) e Incremento Médio Anual (IMA), foram realizados utilizando seguintes fórmulas (ENCINAS et al., 2005):

$$
\text { ICA }=Y_{(t+1)}-Y_{t}
$$

Em que: $I C A=$ incremento corrente anual; $Y=$ dimensão da variável considerada; $t=$ idade

$$
I M A=\frac{Y_{t}}{t_{0}}
$$

Em que: $I M A=$ incremento médio anual; $t_{0}=$ idade a partir do tempo zero; $Y=$ dimensão da variável considerada

\section{RESULTADOS E DISCUSSÃO}

Os dados avaliados para cada tipo de diâmetro com sua estatística descritiva por tratamento (Tabela 2), mostraram o diâmetro médio quadrático (Dg) com valores superiores ao da média aritmética em ambos os tratamentos, comportamento característico de acordo com Campos \& Leite (2017), sendo a medida que mais se relaciona com atributos de povoamentos equiâneos e para o cálculo da área basal e volume. Os diâmetros dominantes (Dd), que foi gerado a partir dos diâmetros das árvores dominantes, foram superiores ao aritmético e quadrático como já esperado, por serem indivíduos mais grossos.

TABELA 2. Estatística descritiva dos tipos de diâmetros por tratamento nos plantios

\begin{tabular}{|c|c|c|c|c|c|c|c|c|c|c|c|c|}
\hline \multirow{2}{*}{$\begin{array}{l}\text { Idade } \\
\text { (anos) }\end{array}$} & \multicolumn{6}{|c|}{ Tratamento 1} & \multicolumn{6}{|c|}{ Tratamento 2} \\
\hline & Mín. & $\overline{d a p}$ & $\mathrm{Dg}$ & Dd & Máx. & CV\% & Mín. & $\overline{d a p}$ & $\mathrm{Dg}$ & Dd & Máx. & CV\% \\
\hline 1 & 2.7 & 7.0 & 7.4 & 8.5 & 14.2 & 26.1 & 1.9 & 6.2 & 6.3 & 8.9 & 15.7 & 36.2 \\
\hline 2 & 5.8 & 11.1 & 11.2 & 12.4 & 17.8 & 19.3 & 2.7 & 8.9 & 9.1 & 9.9 & 16.6 & 25.1 \\
\hline 3 & 8.3 & 14.6 & 14.7 & 16.3 & 20.7 & 14.3 & 6.5 & 12.8 & 12.9 & 14.0 & 17.4 & 14.6 \\
\hline 4 & 13.8 & 18.2 & 18.3 & 20.2 & 23.7 & 9.6 & 8.6 & 15.7 & 15.8 & 17.1 & 20.1 & 10.2 \\
\hline 5 & 14.9 & 19.2 & 19.4 & 20.7 & 26.5 & 13.7 & 10.7 & 18.9 & 19.2 & 22.2 & 22.8 & 8.6 \\
\hline 6 & 16.5 & 21.4 & 21.6 & 22.8 & 28.2 & 11.9 & & & & & & \\
\hline 7 & 18.0 & 23.7 & 23.8 & 25.4 & 29.8 & 10.3 & & & & & & \\
\hline
\end{tabular}
clonais de T. grandis, em Capitão Poço, PA.

Onde: Dg: diâmetro quadrático; Dd: diâmetro dominante, CV \%: coeficiente de variação; Linha pontilhada representa o desbaste aplicado no tratamento $T_{1}$.

Por intermédio do coeficiente de variação, pode-se verificar uma maior variabilidade na variável dap nas idades iniciais, sendo decrescente com o passar da idade, exceto no $T_{1}$ que no quinto ano em teve um aumento de CV\%. Isso está diretamente relacionado com a diminuição da densidade do plantio (desbastes) afetando mais fortemente esta medida.

O teste t student revelou que houve diferença estatística entre os tratamentos ( $p$ valor < 0,05), mostrando crescimento diferenciado em $\mathrm{Hd}$ entre os povoamentos avaliados. A amplitude da variável $H d$ em função das idades (Figura 3), foi semelhante nas três primeiras idades no $T_{1}$ (Figura 3-A), diminuindo a partir do quarto ano, seguido

ENCICLOPÉDIA BIOSFERA, Centro Científico Conhecer - Goiânia, v.15 n.28; p. 947 2018 
de um pequeno aumento a partir do quinto ano, podendo ser justificado pela intervenção na densidade do plantio (desbastes). O mesmo comportamento não ocorreu no $\mathrm{T}_{2}$ (Figura 3-B), onde mostrou-se maior variabilidade nos dados de $\mathrm{Hd}$ nas idades iniciais, sendo decrescente com o aumento da mesma.
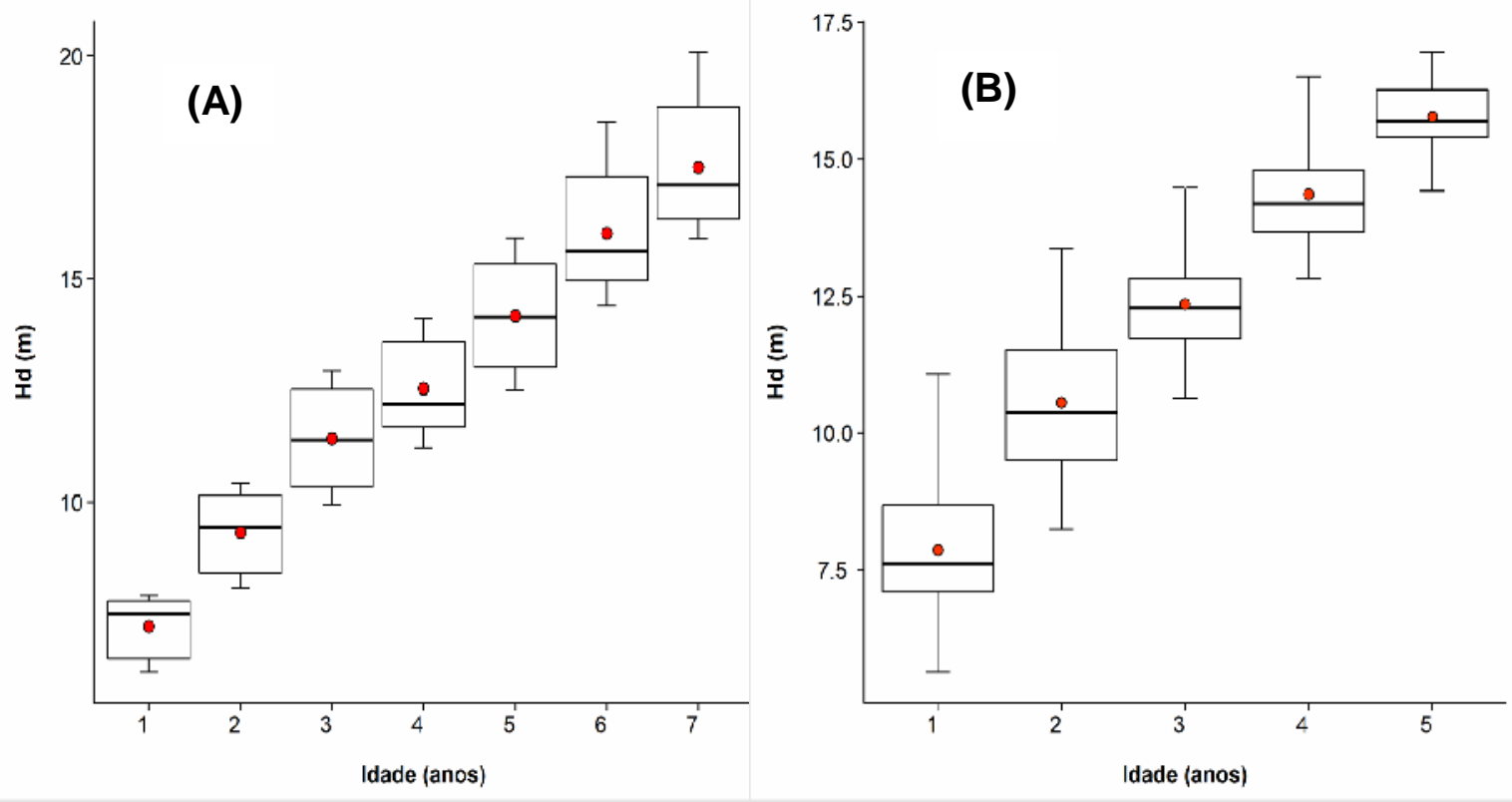

FIGURA 3. Box plot distribuição da variável altura dominante dos tratamentos: $(A) T_{1}$ (plantio 2010) e (B) $\mathrm{T}_{2}$ (plantios 2012 e 2013).

Onde: Linhas horizontais mais grossas representam a mediana; caixas representa o intervalo interquartil ( $1^{\circ}$ e $\left.3^{\circ}\right)$; pontos em vermelho a média aritmética e as linhas verticais os valores extremos. $\mathrm{N}=5\left(\mathrm{~T}_{1}\right)$ e $\mathrm{N}=38\left(\mathrm{~T}_{2}\right)$.

FONTE: Autores (2018).

Ao quarto ano no $T_{1}$, a $H d$ apresentou uma média de $12,5 \mathrm{~m}$, com valores variando entre $11,2 \mathrm{~m}$ e 14,1 $\mathrm{m}$, e com desvio padrão de 1,09 $\mathrm{m}$. O desvio médio em torno da média ficou em $8,7 \%$, representando uma baixa variação. Para $T_{2}$, a altura dominante média foi de $14,2 \mathrm{~m}$, variando entre $12,8 \mathrm{~m}$ e $16 \mathrm{~m}$, com desvio padrão de $0,8 \mathrm{~m}$, indicando uma variação média de $5,6 \%$ em torno da média, ou seja, ainda menos acentuada que a observada no tratamento $\mathrm{T}_{1}$.

Os resultados de ICA e IMA em Hd e dap para os diferentes espaçamentos estão ilustrados na Tabela 3. No monitoramento da altura dominante, verificou-se um superior crescimento no tratamento 2, o que pode estar relacionado ao menor espaçamento destes plantios em comparação ao tratamento 1. Scolforo (2006), comenta que de maneira geral em povoamentos mais densos, há maior crescimento em altura, devido a uma maior competição por luz, levando a um fechamento mais rápido da copa bem como a uma estagnação teórica do crescimento. O contrário ocorreu com a variável diâmetro, em que no tratamento com maior espaçamento $\left(T_{1}\right)$, ocorreu o maior crescimento desta variável, em que a competição é menor e o crescimento em dap é 
mais acentuado, corroborando com Santos et al. (2015), que falaram quanto menor o espaçamento, pode haver maior competição pelo espaço vital de cada e menor será a média aritmética do diâmetro (SCOLFORO, 2006). Dessa forma, a relação entre altura e diâmetro, apresenta uma tendência a ser maior quanto menor for o espaçamento, ou ainda, quanto mais adensado for o plantio (BARTOSZECK et al. 2004; VENDRUSCOLO et al., 2017).

TABELA 3. Incremento médio anual (IMA) e incremento corrente anual (ICA). Média aritmética, IMA e ICA em altura dominante e diâmetro dos tratamentos $T_{1}$ (plantio 2010) e $\mathrm{T}_{2}$ (plantios 2012 e 2013).

\begin{tabular}{|c|c|c|c|c|c|c|}
\hline \multirow{2}{*}{ Idade (anos) } & \multicolumn{3}{|c|}{ Tratamento 1} & \multicolumn{3}{|c|}{ Tratamento 2} \\
\hline & $\overline{H d}$ & ICA Hd & IMA Hd & $\overline{H d}$ & ICA Hd & IMA Hd \\
\hline 1 & $\overline{77,22}$ & & $7,7,2$ & $\bar{~} 7,87$ & & $7,7,9$ \\
\hline 2 & 9,52 & 2,3 & 4,8 & 10,56 & 2,7 & 5,3 \\
\hline 3 & 11,42 & 1,9 & 3,8 & 12,35 & 1,8 & 4,1 \\
\hline 4 & 12,54 & 1,1 & 3,1 & 14,36 & 2,0 & 3,6 \\
\hline 5 & 14,17 & 1,6 & 2,8 & 15,77 & 1,4 & 3,2 \\
\hline 6 & 16,02 & 1,9 & 2,7 & & & \\
\hline 7 & 17,49 & 1,5 & 2,5 & & & \\
\hline Idade (anos) & $\overline{d a p}$ & ICA dap & IMA dap & $\overline{\text { dap }}$ & ICA dap & IMA dap \\
\hline 1 & 7,00 & & $7,7,0$ & 6,20 & & 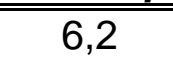 \\
\hline 2 & 11,1 & 4,1 & 5,6 & 8,57 & 2,4 & 4,3 \\
\hline 3 & 14,58 & 3,5 & 4,9 & 12,83 & 4,3 & 4,3 \\
\hline 4 & 18,19 & 3,6 & 4,5 & 15,72 & 2,9 & 3,9 \\
\hline 5 & 19,19 & 1,0 & 3,8 & 18,9 & 3,2 & 3,8 \\
\hline 6 & 21,43 & 2,2 & 3,6 & & & \\
\hline 7 & 23,71 & 2,3 & 3,4 & & & \\
\hline
\end{tabular}

Foi possível verificar no $T_{1}$ uma queda acentuada no incremento em diâmetro no quinto ano, período este, que foi aplicado o desbaste, demonstrando a influência que esta variável sofre com este tratamento silvicultural. Dentre as características utilizadas pela empresa na seleção de indivíduos a serem desbastados, uma foi suprimir aqueles que possuíam galhos grossos (bifurcação) abaixo de $8 \mathrm{~m}$ de altura, sendo, em alguns casos, indivíduos mais grossos, pois estes galhos fazem com que ocorra desaceleração no crescimento em altura e aumento na variável diâmetro, o que pode ser uma justificativa para este comportamento, sendo indivíduos com menor valor comercial. Eloy et al. (2016), comentam que em espaçamentos maiores há formação de galhos mais grossos e copas mais expandidas quando comparados com espaçamentos menores. Em contrapartida, observou-se a baixa interferência que a alteração de densidade entre indivíduos tem sobre a variável $H d$.

$\mathrm{O}$ incremento médio anual em dap variou de 3,4 a $7,0 \mathrm{~cm}$ ano-1 em ambos os tratamentos no período de um a sete anos, superando resultados de crescimento obtidos por Tonini et al. (2009), no estado de Roraima em plantios seminais, onde 
foram observados incrementos em diâmetro abaixo de $3 \mathrm{~cm}$ ano ${ }^{-1}$ nas sete primeiras idades.

Os valores médios de altura dominante nos plantios clonais $(7,22$ a $17,49 \mathrm{~m}) \mathrm{e}$ IMA (2,5 a 7,9 cm ano $\left.{ }^{-1}\right)$ até o sétimo ano, foram superiores aos reportados nos trabalhos de Pelissari el al. (2014) e Tonini et al. (2009), tendo valores de Hd média variando de 1,82 a $16,7 \mathrm{~m}$ e incrementos de 0,67 a 4 m.ano ${ }^{-1}$ em plantios de teca seminais. O comportamento decrescente nas curvas de IMA e ICA em dap e Hd com passar da idade (Figura 4), também foi observado em no trabalho de Tonini et al. (2009).
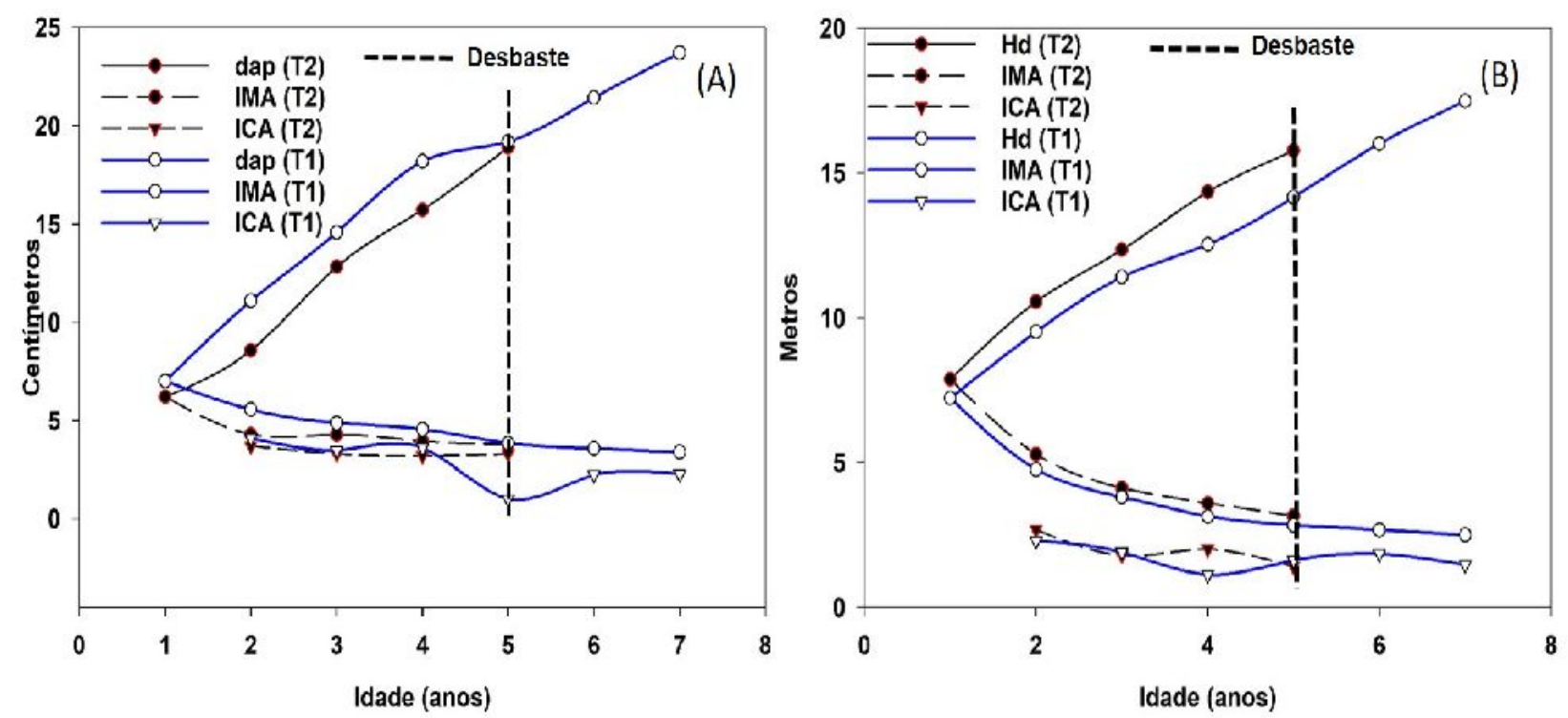

FIGURA 4. Curvas de incremento médio anual (IMA) e incremento corrente anual (ICA). Média aritmética, IMA e ICA em diâmetro dos tratamentos $\mathrm{T}_{1}$ (plantio 2010) e $\mathrm{T}_{2}$ (plantios 2012 e 2013) (A); Média aritmética, IMA e ICA em altura dominante dos tratamentos $T_{1}$ (plantio 2010) e $T_{2}$ (plantios 2012 e 2013) (B).

FONTE: Autores (2018).

Isso demonstra um desempenho característico da espécie com uma redução gradativa na velocidade de crescimento dessas variáveis, provocando a necessidade de intervenções na densidade do povoamento ao longo da rotação a fim de evitar a estagnação do crescimento, sendo povoamentos que requerem mais atenção no monitoramento e condução. Silva et al. (2016) também afirmam que, se não houver intervenção silvicultural, o crescimento e produção, mais especificamente, dos plantios de teca serão afetados de forma não positiva, seja qual for o espaçamento inicial do plantio. Assim como Paiva e Leite (2015), reforçam a relevância do tipo, época, técnica de desbaste nas técnicas de manejo. Além disso, a intervenção na densidade, estimula maior crescimento e produção das árvores, dependendo da intensidade do desbaste e fatores vinculados ao sítio (DAMÁSIO et al. 2015).

Os crescimentos superiores dos plantios clonais de teca em relação aos demais resultados apresentados via semente, podem estar relacionados diretamente à 
utilização de material genético melhorado, mostrando assim a diferença no crescimento quando se utilizam clones em comparação com plantios seminais. Mediante ao conhecimento do crescimento das variáveis estudadas, possibilita obter informações de outras medidas relevantes para um povoamento, como área basal e volume, no caso do diâmetro e avaliação da capacidade produtiva que um local tem de produzir madeira ou outro produto, para $H d$, denominada de índice de sítio (IS).

\section{CONCLUSÃO}

Conclui-se que o espaçamento mais adensado, tratamento $2(3,75 \mathrm{~m} \times 3,75 \mathrm{~m})$, favoreceu o melhor crescimento para a variável $H d$. Já o espaçamento menos adensado, tratamento $1(4 \mathrm{~m} \times 4 \mathrm{~m})$, apresentou maior ritmo de crescimento para a variável dap. Desta forma, pode-se adotar o primeiro ou segundo espaçamento, o que dependerá de qual das variáveis em estudo deve-se priorizar quanto ao seu incremento, possibilitando um planejamento adequado de manejo para os plantios clonais de $T$. grandis no município de Capitão Poço, PA.

\section{REFERÊNCIAS}

ASSMANN, E. The principles of forest yield study. Oxford: Pergamon, 1970.506 p.

BARTOSZECK, A. C. P. S.; MACHADOII, S. do A.; FILHOIII, A. F.; OLIVEIRA, E. B. Dinâmica da relação hipsométrica em função da idade, do sítio e da densidade inicial de povoamentos de bracatinga da região metropolitana de Curitiba, Paraná. Revista Árvore, Viçosa, v. 28, n. 4, p. 517-533, 2004. Disponível em: http://dx.doi.org/10.1590/S0100-67622004000400006.

doi:

$10.1590 / \mathrm{S} 010067622004000400006$

CAMPOS, J. C. C.; LEITE, H. G. Mensuração florestal: Perguntas e respostas. 5ed. Atual. E ampl. UFV. Viçosa, MG. 2017.

DAMÁSIO, M.; da SILVA, F. R.; SANTOS, A. F. A.; NETO, R. M. R. Desbaste seletivo em um povoamento de Tectona grandis implantado em sistema de integração-lavourapecuária-floresta. Biodiversidade, v.14, n.3, p. 74, 2015.

ELOY, E.; da SILVA, D. A.; SCHMIDT, D.; TREVISAN, R.; CARON, B. O.; ELLI, E. F. Effect of planting age and spacing on energy properties of Eucalyptus grandis w. Hill ex maiden. Revista Árvore, Viçosa-MG, v.40, n.4, p.749-758, 2016. Disponível em: http://dx.doi.org/10.1590/0100-67622016000400019. doi: 10.1590/010067622016000400019

ENCINAS, J. I.; da SILVA, G. F.; PINTO, J. R. R. Idade e crescimento dás árvores. Comunicações técnicas florestais, v.7, n.1, 43p, 2005.

GEORGIN, J.; BAZZOTI, R.; PERRANDO, E. Indução ao enraizamento de estacas de teca (Tectona grandis L.f.) Revista Eletrônica em Gestão, Educação e Tecnologia 
Ambiental - V. 18 n. 3 Set-Dez. 2014, p.1246-1256. Disponível em: http://dx.doi.org/10.5902/2236117014537. doi: 10.5902/2236117014537.

HAKAMADA, R. E.; STAPE J. L.; de LEMOS, C. C. Z.; EMANUEL, A.; ALMEIRA, A.; SILVA, L. F. Uniformidade entre árvores durante uma rotação e sua relação com a produtividade em eucalyptus clonais. Cerne, v. 21 n. 3, p. 465-472, 2015. Disponível em: http://dx.doi.org/10.1590/01047760201521031716. doi: 10.1590/01047760201521031716 - Indústria Brasileira de Árvores. Relatório, 2017.

MACHADO, S. do A.; de SOUZA, R. F.; APARECIDO, L. M. T.; RIBEIRO, A.; CZELUSNIAK, B. H. Evolução das variáveis dendrométricas da bracatinga por classe de sítio, Cerne, v. 21, núm. 2, abril-junio, 2015, pp. 199-207. Disponível em: http://dx.doi.org/10.1590/01047760201521021222.

$10.1590 / 01047760201521021222$

MEDEIROS, R. A.; de PAIVA, H. N.; LEITE, H. G. SALLES, T. T.; JÚNIOR, C. A. A.; DÁVILA, F. S. Idade técnica do primeiro desbaste de povoamentos de teca em diferentes espaçamentos. Scientia Forestalis, Piracicaba, v. 45, n. 116, p. 705-716, dez. 2017. Disponível em: http://dx.doi.org/10.18671/scifor.v45n116.11. Doi: 10.18671/scifor.v45n116.11

MIDGLEY, S., MOUNLAMAI, K., FLANAGAN, A., PHENGSOPHA, K. Global market for plantation teak; implications for growers in Lao PDR. ACIAR FST/2010/012: Australian Centre for International Agricultural Research: Canberra. 2015.

MOULIN, J. C.; ARANTES, M. D. C.; de OLIVEIRA, J. G. L.; CAMPINHOS, E.; GOMES, F.; VIDAURRE, G. B. Efeito do Espaçamento, Idade e Irrigação no Volume e Densidade Básica do Eucalipto. Floresta e Ambiente, 24: e00073914, 2017. Disponível em: http://dx.doi.org/10.1590/2179-8087.073914. doi: 10.1590/2179-8087.073914.

do NASCIMENTO, F. A. F; ANDREA N. D.; FIGUEIREDO FILHO, A.; GABRIEL de M. M.; JULIO, E. A. Sistema de crescimento e produção para povoamentos de Pinus taeda na região norte de Santa Catarina. Cerne, Lavras, v. 21, n. 2, p. 235-242, June 2015. Disponível em: http://dx.doi.org/10.1590/01047760201521021494. doi: $10.1590 / 01047760201521021494$

PAIVA, H. N.; LEITE, H. G. Desbastes e desramas em povoamentos de Eucalyptus. In: SCHUMACHER, M. V.; VIERA, M. (Ed.). Silvicultura do eucalipto no Brasil. Santa Maria: Editora UFSM, 2015. p. 83-112.

PElissari, A. LI.; GUIMARÃES, P. P.; BEHLINNG, A.; EBlinNG, Â. A. Cultivo da teca: Características da espécie para implantação e condução de povoamentos florestais. Agrarian Academy, Centro Científico Conhecer - Goiânia, v.1, n.01; p.128, 2014. Disponível em: http://dx.doi.org/10.18677/Agrarian_Academy_2014_011. doi: 10.18677/Agrarian_Academy_2014_011 
PELLICO NETTO, S. P.; BRENA, D. A. Inventário Florestal, Curitiba: Editorado pelos autores, 1997.

dos SANTOS, A. T.; de MATTOS, P. P.; BRAZ, E. M.; ROSOT, N. C. Determinação da época de desbaste pela análise dendrocronológica e morfométrica de Ocotea porosa (Nees \& Mart.) Barroso em povoamento não manejado. Ciência Florestal, Santa Maria, v. 25 , n. 3, p. 699-709, jul.-set., 2015. .

SANTOS, H. G.; JACOMINE, P. K. T.; ANJOS, L. H. C.; OLIVEIRA, V. A.; LUMBRERAS, J. F.; COELHO, M. R.; ALMEIDA, J. A.; CUNHA, T. J. F.; OLIVEIRA, J. B. Sistema Brasileiro de Classificação de Solos. 3. ed. Brasília: Embrapa, 2013. $353 p$.

SCOLFORO, J. R. S. Biometria florestal: Modelos de crescimento e Produção Florestal, Lavras: UFLA/FAEPE, 2006.

SCOLFORO, J. R.. Mensuração florestal: Avaliação da produtividade florestal através da classificação do sítio, Lavras, ESAL/FAEPE, 1993.

DA SILVA, B. N. R; SILVA, L. G. T.; ROCHA, A. M. A.; SAMPAIO, S. M. N. Interação biofísica e do uso da terra na dinâmica da paisagem do município de Capitão Poço-PA, em sistema de informação geográfica. Belém: Embrapa Amazônia Oriental, 1999. 42p.

SILVA, F. R.; e SILVA, V. S. M.; de MIRANDA, S. O.; OLIVEIRA, B. R.; SILVA, J. C. Classificação da produtividade para um plantio de Tectona grandis em Alta Floresta, Mato Grosso. Ambiência Guarapuava, Paraná, v.12 n.4 p. 809 - 823 Set./Dez. 2016. Disponível em: http://dx.doi.org/10.5935/ambiencia.2016.04.04. doi: 10.5935/ambiencia.2016.04.04

SILVA, R. S.; VENDRUSCOLO, D. G. S.; ROCHA, J. R. M.; CHAVES, A. G. S.; SOUZA, H. S.; MOTTA, A. S. Desempenho silvicultural de Tectona grandis L.f. em diferentes espaçamentos em Cáceres, MT. Floresta e Ambiente, Seropédica, v. 23, n. 3, p. 397405, 2016. Disponível em: http://dx.doi.org/10.1590/2179-8087.143015. doi: $10.1590 / 2179-8087.143015$

TONINI, H.; COSTA, M.C.G; SCHWENGBER, L.A. M. Crescimento da teca (Tectona grandis) em reflorestamento na Amazônia setentrional. Pesquisa Florestal Brasileira, Colombo, n. 59, p. 05-14, 2009. Disponível em: http://dx.doi.org/10.4336/2009.pfb.59.05. doi: 10.4336/2009.pfb.59.05

VENDRUSCOLO, D. G. S.; CHAVES, A. G. S.; MEDEIROS, R. A.; SILVA, R. S.; SOUZA, H. S. DRESCHER R.; LEITE, H. G. Estimativa da altura de árvores de Tectona grandis L.f. utilizando regressão e redes neurais artificiais. Nativa, v. 5, n. 1, p. 52-58, jan./fev. 2017. ISSN: 2318-7670 Disponível em: http://dx.doi.org/10.5935/23187670.v05n01a09. doi: 10.5935/2318-7670.v05n01a09 